Marketplace is provided as a service to readers using text and images from the manufacturer, supplier or distributor and does not imply endorsement by Vital. Normal and prudent research should be exercised before purchase or use of any product mentioned.

\section{Banish bad breath}

Dentyl pH has recently launched Exhilaration mouthwash, which has a new triple defence capability. It inhibits VSCs (volatile sulphur compounds), the unpleasant smelling gases generated by oral bacteria and enzymes, well known to be the main cause of bad breath.

The mouthwash combats and removes bacteria which can cause bad breath and plaque and leaves the mouth fresh for up to 18 hours after a single use. In addition, as bacteria cling to the oil phase in the product, when the user rinses out, bacteria and debris are taken with it.

Exhilaration also delivers enduring protection; bacteria are physically removed and do not remain in the mouth as a food source on which the oral bacteria can feed, thereby prolonging the efficacy of the

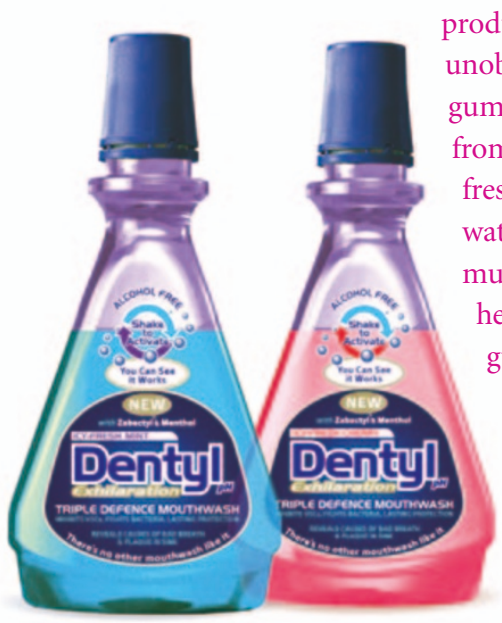
uct. The oil phase provides an film on the teeth and gums, so the mouth is protected from further attack and remains fresher for longer than with water-based products. The formula also includes fluoride to help care for the teeth and gums and strengthen teeth.

Menthol has been added at a fairly high level to ensure an icy-cool in-mouth sensation which increases intensity after use.

\section{A modern alternative}

A new mouth rinse that provides a modern sugar and alcohol free alternative to traditional mouthwash tablets is now available. Pegasus Liquid Mouth Rinse from Pegasus Dental, part of

Piquid
the Astek Group, has launched the new low cost, super concentrated mouthwash with a fresh minty taste. It has also introduced Pegasus strawberry and bubblegum flavoured prophy pastes. The pastes contain only natural flavourings with no fluoride, and special thixotropic handling makes for easy use. The flavours are available in fine, medium and course grain. Each $250 \mathrm{~g}$ tub retails at $£ 4.95$ plus VAT, while each $90 \mathrm{ml}$ bottle of Pegasus Liquid Mouth Rinse, equivalent to 1,100 chairside rinses, is priced at £9.95 plus VAT. marketplace :

\section{Protecting against acid erosion}

A new toothpaste specifically designed to help protect teeth against acid erosion has been introduced by GlaxoSmithKline Consumer Healthcare (GSK).

Sensodyne Pronamel helps to actively micro-harden tooth enamel, making it more resistant to further acid attack, and offers high fluoride availability which may limit the progression of erosion. It also has a very low abrasivity to help limit the amount of softened surface that is removed through the action of tooth brushing.

The product contains potassium nitrate so is specially formulated for people who suffer from dentine hypersensitivity and is also $\mathrm{pH}$ neutral so does not encourage enamel or dentine demineralisation. Sensodyne Pronamel contains the same quality cleaning properties as the rest of the Sensodyne range.

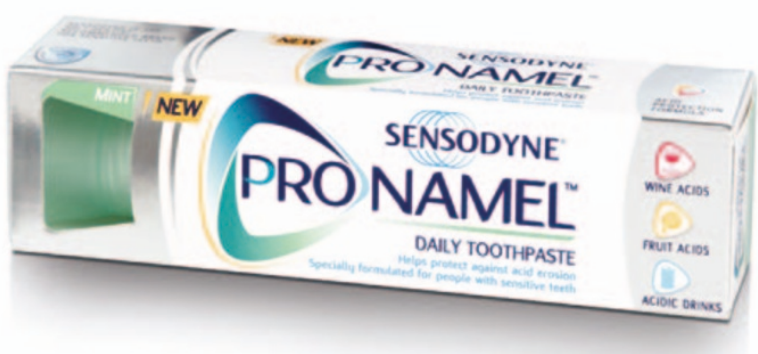

\section{Moistening mouths}

BioXtra produces a wide range of products specially designed to ease the suffering of patients with dry mouth. Superior comfort can be achieved with Mild Antibacterial Toothpaste and Alcohol-free Mouth Rinse for daily hygiene, Non-stick Dental Gum for salivary stimulation, and Moisturising Gel or ultra-convenient Moisturising Gel Mouth spray.

BioXtra Moisturising Gel and Gel Mouth spray can now be NHS prescribed. Patients can also buy BioXtra from pharmacies or by mailorder from Dental Products 2 U Ltd.

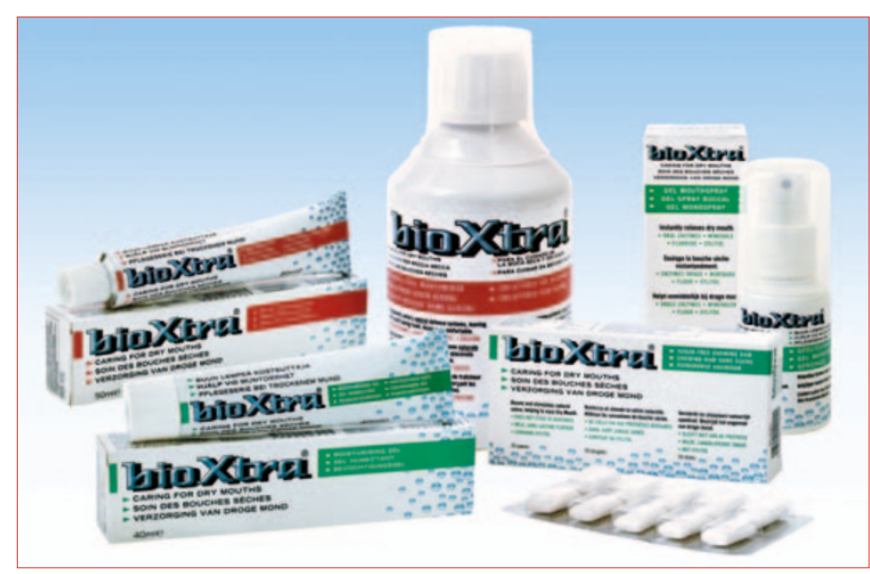

